\title{
De procesos metropolitanos y ciudades sustentables
}

\section{Metropolitan processes and sustainable cities}

\author{
GraizBord, Boris (20I4), METRÓPOLIS: ESTRUCTURA URBANA, \\ MEdio AMBIENTE Y POLÍtICA PÚBlICA, El Colegio de MÉxico, \\ MÉXICO, 6I 8 PP., ISBN: 978-607-462-659-9
}

Me gustaría aprovechar esta ocasión para reconocer algunos méritos del editor y coordinador de este libro, Boris Graizbord. Este trabajo constituye uno más que se suma a su enorme trayectoria en el campo de los estudios urbano-ambientales en México. Graizbord es pionero en la investigación sobre este tema en nuestro país. En 1993, publicó, junto con María Eugenia Negrete y Crescencio Ruiz Chiapetto, uno de sus primeros libros Población, espacio y medio ambiente en la ZMCM. ${ }^{\mathrm{I}}$ Desde entonces, ha escrito numerosas obras, casi un centenar, entre libros, capítulos y artículos. Además de su actividad como investigador, quizá lo más entrańable para quien escribe, es su lucidez y su entusiasmo como maestro. Varias generaciones de alumnos de El Colegio de México, El Instituto "Dr. José Ma. Luis Mora”, la Universidad Nacional Autónoma de México y El Colegio Mexiquense se han formado con alguien que conoce bien la geografía y economía urbana, así como las ciencias ambientales vinculadas con la ciudad y la política urbana y ambiental.

A través de esta generosa labor cotidiana, que es la enseñanza, Graizbord ha inspirado a unos y convencido a otros, como es mi caso, a base de repetición pedagógica, sobre la importancia de estudiar la sostenibilidad y el problema ambiental en las ciudades. Finalmente, otra veta de su trabajo es su cargo como coordinador del Programa de Estudios Avanzados en Desarrollo Sustentable y Medio Ambiente (LEAD-México) desde 1995 y hasta hoy. Por medio de este programa, ha participado en la formación de líderes, algunos de ellos se desempeñan como servidores públicos en las diferentes esferas de la Administración Pública. Mientras que otros son activistas sociales, quienes trabajan día a día para enfrentar el problema ambiental en las ciudades.

También quisiera aprovechar esta oportunidad para reconocer al Centro de Estudios Demográficos, Urbanos y Ambientales de El Colegio

${ }^{1}$ Negrete, M., B. Graizbord, y C. Ruiz (1993), Población, espacio y medio ambiente en la ZMCM, El Colegio de México, México. 
de México que ha editado el libro. Este centro cuenta con más de 50 años, desde su fundación en 1964, de experiencia en la formación y difusión dentro de las áreas temáticas que se desarrollan en esta obra.

A lo largo de sus 17 capítulos y un anexo, Metrópolis: estructura urbana, medio ambiente y política pública, el autor explora la realidad de la metrópolis actual en México. La época histórica se sitúa en plena globalización, la cual empieza a ser evidente en nuestro país a finales de la década de los ochenta y principios de los noventa, lapso en el que la ciudad compacta y monocéntrica del periodo del capitalismo industrial da paso a una ciudad más extendida, poblada y policéntrica. Estas características producen ventajas, pero también múltiples problemas para el buen funcionamiento de la ciudad y la calidad de vida de los citadinos. Ante esta situación, la teoría urbana se enfrenta a la necesidad de entender y organizar los extremadamente variados procesos que tienen lugar en nuestras ciudades. También, se presenta la necesidad de clarificar y asumir los principios éticos y políticos, entiéndase básicamente, la sustentabilidad ambiental, la inclusión social, el desarrollo económico o la gobernanza, los cuales debieran guiar, a través de la planeación urbana, la construcción de las ciudades.

Graizbord plantea tres preguntas que organizan el material del libro. La primera consiste en indagar sobre las características espaciales de la urbanización. La segunda explora cuáles podrían ser los criterios urbanos para transitar a formas ambientalmente más sostenibles. Finalmente, en la tercera reflexiona sobre cuáles serían algunos de los criterios para mejorar el gobierno de las metrópolis, enfocándose en el problema ambiental. Con base en estas preguntas, el libro se organiza en tres apartados: "estructura urbana", "medio ambiente" y "política pública".

Este escrito heterogéneo reúne a investigadores ya consolidados y a otros que están en proceso de serlo. Algunos de los capítulos provienen de investigaciones de tesis doctorales; por esta razón, además de interesantes y bien planteados, proporcionan mucha información empírica para quienes se dedican a este campo de estudio y disciplinas afines. Otros capítulos son resultado de investigaciones pequeñas, pero sumamente valiosas y necesarias para generar conocimiento y debate. El libro cuenta además con propuestas de política y gestión urbana. Todo lo anterior, lo convierten en un texto con mucha riqueza de contenido que, sin duda, será una herramienta útil para la investigación, la gestión urbana-ambiental y la docencia. 


\section{Primera parte: Estructura urbana}

El propósito de esta sección es explorar, a través de nueve capítulos, la estructura y función urbana de la metrópolis actual. La atención se centra en la Zona Metropolitana de la Ciudad de México, pues se analiza en siete capítulos; sólo uno, el de Garrocho y Álvarez, toma como caso de estudio a la Zona Metropolitana de Toluca.

Boris Graizbord es el autor del primer capítulo: "Calidad de vida urbana: ¿sinónimo de ciudad sustentable?”. En él, parte de las limitaciones que enfrenta la configuración de la Administración Pública de nuestro país para poder plantear acciones que respondan al interés del desarrollo urbano sustentable. Ante esta situación, propone un marco de referencia que podría ser útil para ello. Se trata de la noción de calidad de vida que, como explica Graizbord, presenta buenas oportunidades para ser un marco político de referencia para el desarrollo urbano sostenible.

De la misma forma, el autor recupera el modelo de Myers (1994), el cual vincula el concepto de calidad de vida con el de la planeación urbana. Éste muestra las interacciones entre el crecimiento urbano y múltiples variables por ejemplo: el costo de la vida, la calidad ambiental, el empleo y las infraestructuras. Las relaciones son positivas y negativas, según sea la diada. Cuando existe un efecto negativo es necesario intervenir para romper con esta forma de interacción que afecta la calidad de vida de los citadinos.

El segundo capítulo, a cargo de Adrián Guillermo Aguilar y Flor López, se titula: "La periurbanización y los retos de su organización territorial". Este texto representa un enorme aporte al análisis de la metrópolis actual, el cual, a diferencia de otras temáticas, no ha sido suficientemente estudiado por los urbanistas en México, y tampoco se ve reflejado en la agenda de la política territorial. A través de la lectura del capítulo, los autores dejan ver las imágenes del espacio periurbano de las grandes metrópolis, las cuales remiten a lo lejano en relación con el centro de la ciudad, a los bordes, no sólo en términos de lo geográfico sino también en lo social. Son espacios donde el principio del derecho a la ciudad adquiere un significado y una necesidad urgentes.

Los autores insisten en la necesidad de entender primero qué es la periferia urbana, en cuanto a sus procesos, actores y transformaciones recientes, e ilustran con suficiente información que la periferia escapa a los estereotipos, como los espacios de la segregación y pobreza; pues más allá de esta realidad se observan múltiples tipos de espacios según su condición sociocultural, económica, política y ambiental. A partir de la reflexión teórica y el análisis empírico de la Ciudad de México, Aguilar y 
López adelantan siete sugerencias para responder al reto de la planeación territorial en las periferias de las grandes metrópolis.

En el tercer capítulo, "Estructura urbana del АмСм: un enfoque ecológico”, Raúl Lemus nos presenta un análisis detallado de la organización interna del área metropolitana de la Ciudad de México para dos cortes en el tiempo, 1990 y 2000. Con base en los datos de uso del suelo, dividido en habitacional, mixto e industrial, reconstruye la estructura urbana. El trabajo de recopilación y reconstrucción de información es enorme, lo cual se refleja en la riqueza de la información que muestran sus mapas. El propósito central de la investigación es averiguar si la estructura de la Ciudad de México es coherente con alguno de los modelos clásicos de la ecología urbana, ya sea el de círculos concéntricos, el de sectores o el de núcleos múltiples. Los resultados indican que no se ajusta a ninguno de éstos, sino que se da una combinación. En todo caso, sus resultados dan lugar a pensar en la necesidad de actualizar los conceptos y teorías de la Ecología urbana, para dar cuenta de la realidad de la estructura urbana actual.

Los siguientes tres capítulos abordan un tema clave en la economía urbana: la ciudad global (Sassen) o el sistema mundial de ciudades (Gordon y Richardson). El primer estudio, "Análisis comparativo de la estructura económica de los distritos centrales de dos ciudades mundiales: Manhattan, Nueva York y delegaciones centrales de la Ciudad de México", corresponde a Fermín Cruz. El argumento es que en las ciudades llamadas globales se concentran los servicios que requieren una mayor calificación de mano de obra. Según Sassen (1991), estas ciudades son Nueva York, Londres y Tokio. El objetivo del autor es comparar una de este rango, Nueva York, con la Ciudad de México. Los resultados indican que la globalización y sus conceptos asociados han propiciado cambios en la estructura productiva y su localización; sin embargo, las diferencias observadas entre las dos realidades estudiadas apuntan a la necesidad de precisar y distanciarse de las teorizaciones elaboradas para procesos que se observan más claramente en las ciudades del norte global.

En el siguiente capítulo, "La provisión de servicios de internet en México: ¿Tiene un dominio nacional de este servicio la ZMCM?”, Rubén Garnica cuestiona la idea de las ciudades globales y plantea que no hay una entidad que se pueda considerar como la economía global, sino que se trata de circuitos que funcionan a diferentes niveles: local, regional y mundial. Con base en este argumento, Garnica estudia los servicios de internet en la Ciudad de México, para entenderla como nodo nacional. Los resultados, para el caso del internet, muestran que no existe un dominio o hegemonía de la Ciudad de México, sino un sistema regional conformado por las tres grandes ciudades del país: Ciudad de México, 
Guadalajara y Monterrey. Entonces, el internet se localiza en las grandes ciudades, como lo indican otras investigaciones, pero no ha generado nuevos sistemas de ciudades, sino que ha reforzado el mismo sistema regional construido en el periodo de la industrialización y metropolización de la posguerra.

Finalmente, Alberto Paulino, en "La conectividad aérea de la Ciudad de México dentro del sistema mundial de ciudades", ofrece información adicional y complementaria a la de los dos autores anteriores, para interpretar a la Ciudad de México con base en la idea de la ciudad mundial. De acuerdo con el autor, la conectividad aérea es una condición necesaria para que una ciudad sea viable dentro del contexto de la globalización. El autor realiza el análisis empírico con los datos del total de vuelos, el volumen de carga y el total de pasajeros hacia y desde la Ciudad de México para el 2007. Los resultados muestran que la Ciudad de México sí es una ciudad mundial, la cual se encuentra muy asociada con las primeras siete categorías de la jerarquía de ciudades mundiales. Aunque su relación es notablemente más intensa con los Estados Unidos, 10 ciudades, en comparación con seis ciudades europeas y seis sudamericanas.

El estudio de Rocío González, "Patrones espaciales y decisiones de movilidad residencial en la Zona Metropolitana de la Ciudad de México", representa un cambio de tema. El propósito de su capítulo es conocer los patrones espaciales de movilidad residencial en la ZMCM entre 1995 y 2000, así como los atributos de las personas que realizaron estos movimientos. Resulta interesante, entre todos sus hallazgos, saber que la movilidad residencial intraurbana es un fenómeno importante para entender la estructura y funcionamiento de la ciudad en el corto y mediano plazo. La autora encontró que, del total de cambios que se registraron, la movilidad residencial representa 30\%, mientras que la migración corresponde a 70 por ciento.

El sector de la ZMCM más dinámico es el de los municipios del Estado de México (este-norte). El factor vivienda tiene una gran relevancia para explicar la movilidad residencial, pues la población que realizó un movimiento buscó obtener una vivienda propia. Asimismo, la autora ratificó que los patrones espaciales de la movilidad residencial responden a múltiples variables; más allá de la cercanía al trabajo, también son importantes algunas variables que se asocian al ciclo de vida (edad), el sexo y las características de la comercialización de las viviendas.

Emelina Nava y Jaime Ramírez presentan el capítulo: "Movilidad sustentable: consideraciones sobre el tiempo de desplazamiento de viaje en el АмСм". De acuerdo con los autores, una política urbana de movilidad sustentable debe basarse en el estudio serio de la situación de las prácticas de desplazamiento de sus residentes. En este capítulo, Nava y 
Ramírez trabajan en este sentido, y elaboran un análisis de los desplazamientos cotidianos de los habitantes del área metropolitana de la Ciudad de México. Estudian dos variables, la distancia lineal y el tiempo de desplazamiento, con respecto al propósito de los viajes: productivos, de consumo y sociales. La fuente de datos para realizar el estudio es la encuesta de origen y destino de 1994. Este estudio ofrece una enorme cantidad de información sobre los desplazamientos de los citadinos. De una forma muy general, podemos mencionar, por ejemplo, que el promedio de tiempo de desplazamiento de los residentes del AMCM es de una hora con cuarenta y tres minutos. Otro resultado importante es que la valoración del tiempo y la distancia es más importante cuando se trata de los viajes productivos y sociales, en comparación con los de consumo.

A Carlos Garrocho y José Antonio Álvarez corresponde el capítulo titulado: "El patrón espacial de un sistema bancario en el territorio intrametropolitano: aglomeración, dispersión, aleatoridad y co-localización”. El principal objetivo de este trabajo es de naturaleza metodológica; consiste en utilizar la función $K$ para medir la aglomeración, dispersión, aleatorieded y co-localización de las sucursales bancarias. Anteriormente, en un estudio de 2010, los autores habían utilizado la técnica del "vecino más cercano" para estudiar el patrón de localización espacial de las sucursales bancarias en la zona metropolitana de Toluca.

Las limitaciones que encontraron los animaron a indagar sobre la función $K$. Su trabajo muestra que el método presenta múltiples ventajas para caracterizar los patrones espaciales de puntos, entre éstas destaca la posibilidad de realizar análisis a diversas escalas de manera simultánea, lo que no puede hacerse con la técnica del "vecino más cercano". Otra de las ventajas es que sus resultados no se ven afectados por la forma o el tamaño del área de estudio. Finalmente, los autores explican que esta metodología también es más robusta en términos estadísticos. No obstante, la función $K$ también presenta desventajas. En todo caso, como se analiza en uno de sus apartados, el método del "vecino más cercano" es complementario con la función $K$, pues cada uno genera información distinta y útil para caracterizar con mayor exactitud y confiabilidad los patrones de localización de las sucursales bancarias o cualquier otro tipo de unidades económicas.

\section{Segunda parte: Medio ambiente}

La segunda parte contiene cinco capítulos, en los cuales se reflexiona, a través de diversos temas, sobre el problema ambiental. El primer capítulo, a cargo de Rigoberto García, se titula: "Los usos de la energía como factor de diferenciación social: un análisis en los ámbitos urbano y rural 
de México". Este trabajo se inserta en una línea de investigación que se ha venido desarrollando en las últimas dos décadas, la cual se relaciona con el papel que tiene la energía para mejorar la calidad de vida de la población. Por lo general, según explica el autor, los estudios se centran en la relación entre el uso de energía y la pobreza en el ámbito rural.

Sin negar la importancia de éstos, García se enfoca en analizar lo que sucede en el ámbito urbano. La investigación determina que la transformación histórica urbana e industrial de la sociedad mexicana de la posguerra provocó el aumento exponencial de las necesidades energéticas de la población. Además, el uso de la leña y el queroseno fue sustituido por energéticos de mayor calidad, es decir, el gas licuado y la electricidad. Los resultados de esta investigación cuestionan la importancia que se le atribuye en otros estudios, a la variable rural $v$ s. urbana, para determinar la diferenciación social según el uso de la energía.

El segundo capítulo, "El consumo de energía de los hogares en México: presión ambiental y desarrollo sostenible. Una aproximación a través de los datos de la ENIGH 2006", corresponde a Adriana Larralde y también aborda el tema del consumo energético. El análisis parte del principio político y ético de que sin una restricción del consumo (excesivo) y del sistema de valores que lo animan, el desarrollo sostenible no es alcanzable. Para medir el consumo de la población se utiliza la variable "gasto en requerimientos de energía". El objetivo del trabajo es analizar la relación entre el consumo de energía y los diferentes tipos de hogares en México. Este ejercicio se lleva a cabo con los datos agregados de la Encuesta de Ingresos y Gastos de los Hogares. Los resultados indican que la variable más explicativa del nivel de consumo de los hogares es el ingreso. Asimismo, el consumo de energía eléctrica refleja de forma más directa la brecha de desigualdad. También se obtienen algunos resultados interesantes en relación con múltiples variables asociadas a la vivienda. Finalmente, se encontró que los hogares urbanos consumen más energía que los rurales, aunque el coeficiente de correlación no es alto.

El tercer capítulo fue escrito por María Eugenia García y se titula "Sustentabilidad urbana y áreas verdes en la ciudad de México". Esta investigación se centra en el análisis de las áreas verdes en el espacio intraurbano de la Ciudad de México. La ciudad, por su carácter concentrado, es una gran consumidora de recursos naturales y energía, y por lo tanto, difícilmente sustentables. No obstante, sí puede adquirir importantes atributos en el logro de la sustentabilidad; una de estas vetas se encuentra en las áreas verdes urbanas. El argumento central de su estudio es que éstas desempeñan un papel importante en el desarrollo de una conciencia ambiental, dada su cercanía y evidencia inmediata. 
La autora elabora una encuesta en el Bosque de Chapultepec, con el propósito de analizar la relación que tiene la población con las áreas verdes de la ciudad y con el concepto de sustentabilidad. La información obtenida es interesante y variada. A fin de cuentas, se puede decir que la población entrevistada no percibe que las áreas verdes tengan una importancia ambiental.

El cuarto capítulo, "Agua para uso público-urbano y sustentabilidad ambiental en México", de la autoría de Nicolás Sisto, trata sobre el suministro de agua en la ciudad, uno de los problemas ambientales más frecuentes que experimentan las ciudades en México. El autor se enfoca en dos casos de estudio: la zona metropolitana de La Laguna y la zona metropolitana de Monterrey, ambas ubicadas en el norte del país. Parte del problema que surge de la relación entre el crecimiento de las ciudades y el crecimiento de la demanda de agua en un contexto de recursos limitados. Sisto se centra en analizar dos soluciones de política pública: 1. el costo del agua para los usuarios y 2 . la intensificación del aprovechamiento de fuentes existentes, incluyendo las aguas residuales tratadas. Una aportación clave del trabajo es el diseño de una metodología que establece la relación entre el manejo de la demanda y la oferta existente del recurso, la cual incluye múltiples variables y técnicas de análisis que el lector especializado podrá apreciar.

Las conclusiones son alentadoras, en el sentido de que el crecimiento de las áreas urbanas no es necesariamente incompatible con la sustentabilidad en el suministro del agua. El autor alerta sobre la necesidad de contar con un enfoque integral que considere simultáneamente todos los elementos del sistema. Además, nos dice que no existe un procedimiento "estándar", pues cada cuenca y cada área metropolitana presentan una combinación única de características; por lo anterior, se requiere un plan de manejo hecho a la medida de las particularidades de cada caso.

En el último capítulo de esta sección, Fernando Aragón presenta "Urbanización y vulnerabilidad: las inundaciones en la interfase periurbana de la Ciudad de México". En junio del 2000, Valle de Chalco, una zona habitada por una población de bajos recursos, sufrió una gran inundación de aguas negras ( 80 hectáreas). La catástrofe se debió a la ruptura del canal La Compañía. En esta investigación, Aragón pretende dar una respuesta a las causas de la inundación en Valle de Chalco. Su explicación parte de un enfoque histórico del cambio socioambiental; igualmente, prioriza el papel de los actores e instituciones en la construcción del riesgo. El autor desarrolla tres aspectos para analizar este desastre. En primer lugar, estudia la inundación en la interfase periurbana con una perspectiva sociohistórica. Expone las causas de la vulnerabilidad que se originaron tanto en los espacios urbanos como en los rurales. El segundo 
aspecto que aborda Aragón es la relación entre la urbanización, las políticas que han sido instrumentadas en el pasado y la generación del riesgo de inundación. Finalmente, el tercer aspecto consiste en el estudio de la respuesta de la política actual y el manejo del canal La Compañía. La situación actual de La Compañía es un ejemplo de la combinación de las transformaciones físicas del ambiente hechas por el hombre. La Compañía, según el autor, es un riesgo construido por el hombre.

\section{Tercera parte: Política pública}

Esta sección está conformada por tres capítulos y un apéndice. El primero constituye una investigación realizada por Jesús Arroyo y David Rodríguez, titulada "Gobernanza local urbana y medio ambiente en México". El objetivo de los autores es explorar cuáles son las capacidades de los gobiernos de los municipios urbanos de todo el país para resolver los problemas ambientales. Básicamente, se enfocan en el tema de la participación ciudadana, dada su relevancia en el discurso de la gobernanza. El trabajo ofrece un panorama exhaustivo, realizado con base en los datos de los censos de 2000 y 2004 de todos los municipios en México de la Sedesol. Los resultados de los autores ilustran que, si bien las responsabilidades de los municipios y sus atribuciones en materia ambiental se han multiplicado durante las últimas décadas, las capacidades de los ayuntamientos aún son incipientes. Los problemas medioambientales ocupan los últimos lugares entre las prioridades de uso de los recursos técnicos, financieros y humanos para los municipios urbanos.

El segundo capítulo, "Estructura y dinámica urbana en Mexicali: retos para una planeación sustentable", fue elaborado por Arturo Ranfla, Osvaldo Leyva y Rosa Imelda Rojas. En él se aborda una preocupación central de los estudiosos de la ciudad y el problema ambiental: la relación entre forma urbana y sostenibilidad ambiental. El crecimiento extensivo y disperso del área urbana vinculado con el uso del automóvil supone un mayor consumo energético y, en consecuencia, un mayor deterioro ambiental. La integración urbana es el argumento clave para el análisis. Ésta supone que la situación ideal para la ciudad sería la concentración-articulación entre la actividad económica y residencial, con el propósito de minimizar los desplazamientos y, por ende, el consumo energético. Los resultados ilustran la situación actual de la forma y el funcionamiento urbano según los sectores de la ciudad. En general, se observa un nivel bajo de integración. Los desplazamientos de la población y la interacción entre zonas es alta y, en consecuencia, se infiere que también el deterioro ambiental. Esta situación llama la atención sobre la necesidad de planifi- 
car la ciudad, en cuanto a los usos del suelo, para mejorar sus condiciones de sustentabilidad.

En el tercer capítulo, Boris Graizbord, José Luis González, Adriana Larralde y Rocío González presentan el procedimiento metodológico que se utilizó para elaborar la Agenda de sustentabilidad ambiental para la Zona Metropolitana del Valle de México. Dicha metodología podría resultar de utilidad para otras zonas metropolitanas del país. Uno de los elementos interesantes es el proceso de identificación de los problemas ambientales urgentes, los cuales deben ser abordados de forma integral para toda la zona. La primera etapa en la identificación proviene de la revisión de los diagnósticos de los programas gubernamentales existentes en cada uno de los estados que abarca la zona. La segunda etapa consiste en la realización de entrevistas a informantes clave, tanto del sector público como del académico y el social, sobre los problemas ambientales y los retos para la planeación ambiental. Es importante destacar que la definición del problema ambiental y el nivel de prioridad que le asignan los entrevistados para su inclusión en una agenda política, va más allá del lugar (municjipio, estado) que habitan o representan; pues es fundamental la adscripción social de cada informante. En este sentido, es necesario complementar los análisis técnicos de la zona, con la percepción de los actores y, la voluntad política que muestran para enfrentar cada uno de ellos.

Por último, en el apéndice, Jaime Eduardo García presenta "El Programa Nacional de Auditoría Ambiental de México y la Certificación de Calidad Ambiental Municipal". El autor explica en qué consisten las Auditorías Ambientales, un instrumento de política ambiental que promueve la Procuraduría Federal de Protección al Ambiente. Dicho instrumento refleja las dificultades que enfrenta el Estado ante el problema ambiental a nivel del gobierno local y los esfuerzos que se realizan para solventarlo. El autor se enfoca en dos tareas estratégicas de los municipios: el manejo de residuos sólidos y el tratamiento de aguas residuales. La certificación voluntaria para el mejoramiento del desempeño ambiental municipal, a través de las auditorías, es una medida adicional y complementaria a las normas existentes (la Ley General para la Prevención y Gestión Integral de los Residuos, la Ley de Aguas y las Normas Oficiales Mexicanas). La mayoría de estas certificaciones se basan en estándares internacionales y, al parecer, han tenido buenos resultados como estrategias de planeación para algunos municipios donde se han desarrollado las auditorías.

Hasta aquí les dejo las generalidades de un libro grande, Metrópolis: estructura urbana, medio ambiente y política pública, y los invito a descu- 
brir capítulo a capítulo la riqueza de contenido que cada uno de los autores nos presentan.

Recibido: 28 de agosto de 2015. Aceptado: 10 de septiembre de 2015.

Adriana Larralde-Corona El Colegio Mexiquense, A. C. Correo-e: alarraldecmq.edu.mx

Adriana Larralde-Corona. Doctora en ciencia social con especialidad en sociología por El Colegio de México. Actualmente es profesora-investigadora en El Colegio Mexiquense, A. C. Es miembro del Sistema Nacional de Investigadores, nivel I y perfil Promep-SEP. Sus líneas de investigación son ciudad dispersa y relaciones campo-ciudad y desarrollo rural sustentable. Entre sus publicaciones más recientes se encuentran: "El consumo de energía de los hogares en México: presión ambiental y desarrollo sostenible. Una aproximación a través de los datos de la ENIGH 2006”, en Boris Graizbord (ed.), Metrópolis: estructura urbana, medio ambiente y política pública, El Colegio de México, México, pp. 381-403 (2014); en coautoría: "Teletrabajo: una estrategia de mitigación de GEI para el Área Metropolitana de la Ciudad de México", en Giourgulli Saucedo, Silvia E. y Vicente Ugalde (coords.), Gobierno, territorio y población: las políticas públicas en la mira, El Colegio de México, México, pp. 645-689 (2014); "Conceptualización y medición de lo rural: Una propuesta para clasificar el espacio rural en México", Consejo Nacional de Población. La situación demográfica de México, Consejo Nacional de Población, México, pp. 141-157 (2013). 